\title{
Relationship between n-3 Polyunsaturated Fatty Acids and Extent of Vessel Disease in Patients with ST Elevation Myocardial Infarction
}

\author{
Takenobu Shimada, ${ }^{1}$ MD, Kazushige Kadota, ${ }^{1}$ MD, Haruki Eguchi, ${ }^{1}$ MD, Kohei Osakada,${ }^{1}$ MD, \\ Akimune Kuwayama, ${ }^{1}$ MD, Masanobu Ohya, ${ }^{1}$ MD, Katsuya Miura, ${ }^{1}$ MD, Hidewo Amano, ${ }^{1}$ MD, \\ Shunsuke Kubo, ${ }^{1}$ MD, Masatomo Ozaki ${ }^{1}$ MD, Yusuke Hyodo, ${ }^{1}$ MD, Mitsuru Yoshino, ${ }^{1}$ MD, \\ Koshi Miyake, ${ }^{1}$ MD, Yuichi Kawase, ${ }^{1}$ MD, Noriyuki Ohashi, ${ }^{1}$ MD, Suguru Otsuru, ${ }^{1}$ MD, \\ Hiroshi Tasaka, ${ }^{1}$ MD, Seiji Habara, ${ }^{1}$ MD, Takeshi Tada, ${ }^{1}$ MD, Hiroyuki Tanaka, ${ }^{1}$ MD, \\ Takeshi Maruo, ${ }^{1}$ MD, Yasushi Fuku, ${ }^{1}$ MD, Harumi Katoh, ${ }^{1}$ MD, Satoki Fujii, ${ }^{1}$ MD, \\ Tsuyoshi Goto, ${ }^{1}$ MD and Kazuaki Mitsudo, ${ }^{1}$ MD
}

\begin{abstract}
Summary
A relationship between serum polyunsaturated fatty acids (PUFAs) and cardiovascular disease has been reported; however, the existence of a relationship between serum PUFAs and extent of vessel disease (VD) in patients with ST elevation myocardial infarction (STEMI) remains unclear.

Between July 2011 and June 2015, 866 consecutive STEMI patients underwent emergent percutaneous coronary intervention, 507 of whom were enrolled and classified into three groups according to the initial angiograms: 1VD, 294 patients; 2VD, 110 patients; and 3VD/left main trunk disease (LMTD), 103 patients. Serum levels of PUFAs, including eicosapentaenoic acid (EPA), docosahexaenoic acid (DHA), and arachidonic acid, and other laboratory data during hospitalization were evaluated.

The serum EPA level in the 3VD/LMTD group was significantly lower than that in the $1 \mathrm{VD}$ group $(55.5 \pm$ 22.1 versus $66.2 \pm 28.7, P=0.002)$ and was slightly lower than that in the $2 \mathrm{VD}$ group $(55.5 \pm 22.1$ versus 65.2 $\pm 28.9, P=0.0167$ ). Multivariate adjustment analysis revealed that age $\geq 70$ years (odds ratio, 1.72 ; $95 \%$ confidence interval, 1.03-2.89; $P=0.038$ ) and a low serum EPA level (odds ratio, 0.98; 95\% confidence interval, 0.99-1.00; $P=0.023$ ) were independent risk factors for 3VD/LMTD, while a low serum DHA level was not.

A low serum EPA level may be more strongly related than a low serum DHA level to the extent of VD in STEMI patients. Age $\geq 70$ years and a low serum EPA level may be independent risk factors for 3VD/LMTD.

(Int Heart J 2017; 58: 868-873)

Key words: Eicosapentaenoic acid, Docosahexaenoic acid, Mulivessel disease, Acute coronary syndrome, Cardiovascular disease
\end{abstract}

A $\mathrm{n}$ association between fish intake and risk of cardiovascular disease has been demonstrated in several epidemiological studies and clinical trials. ${ }^{1-4)}$ Fish oil includes n-3 polyunsaturated fatty acids (PUFAs), among which eicosapentaenoic acid (EPA) and docosahexaenoic acid (DHA) are known to be inversely associated with the extent of atherosclerosis, cardiovascular events, and mortality. ${ }^{5-12)}$

\section{Editorial p.837}

Recent studies have demonstrated a direct relationship between n-3 PUFAs and the extent of coronary atherosclerosis. Amano, et al showed that low levels of n-3 PUFAs are associated with lipid-rich plaques detected by using integrated backscatter intravascular ultrasound,${ }^{13)}$ and Urabe, et al reported that a low serum EPA level is associated with the presence and extent of high-risk plaques detected by coronary computed tomography angiography in patients receiving statin therapy. ${ }^{14)}$ However, little is known about the relationship between n-3 PUFAs and coronary atherosclerosis in patients with acute coronary syndrome, especially in patients with ST elevation myocardial infarction (STEMI).

We investigated the relationship between serum PUFAs, namely EPA, DHA, and arachidonic acid (AA), and the extent of vessel disease (VD) as a measure of coronary atherosclerosis in STEMI patients.

From the ${ }^{1}$ Department of Cardiology, Kurashiki Central Hospital, Kurashiki, Japan.

Address for correspondence: Takenobu Shimada, MD, Department of Cardiology, Kurashiki Central Hospital, 1-1-1 Miwa, Kurashiki, Okayama 710-8602, Japan. E-mail: takeboyism@gmail.com

Received for publication November 9, 2016. Revised and accepted January 27, 2017.

Released in advance online on J-STAGE November 17, 2017.

doi: 10.1536/ihj.16-565

All rights reserved by the International Heart Journal Association. 


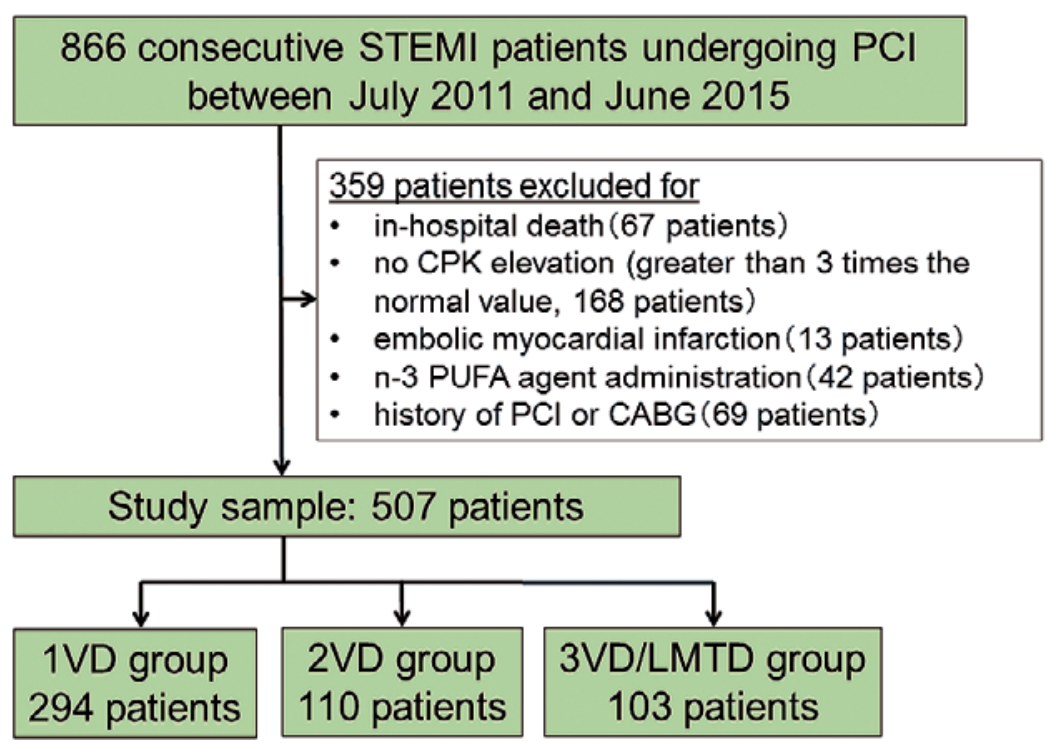

Figure 1. Study flow chart. STEMI indicates ST elevation myocardial infarction; PCI, percutaneous coronary intervention; CABG, coronary artery bypass grafting, PUFA, polyunsaturated fatty acid; VD, vessel disease; and LMTD, left main trunk disease.

\section{Methods}

Study sample and study protocol: This study was designed as a single center, cross-sectional study to assess the relationship between n-3 PUFAs and the extent of VD as a measure of atherosclerosis in STEMI patients. The diagnosis of STEMI was based on the Universal Definition of Myocardial Infarction, ${ }^{15}$ including a rise of cardiac biomarker values with symptoms of ischemia and new or presumably new significant ST segment changes. The exclusion criteria were n-3 PUFA agent administration, no severe cardiac damage (creatine phosphokinase level remaining not greater than 3 times the upper limit of the normal range), embolic myocardial infarction, history of percutaneous coronary intervention or coronary artery bypass graft surgery, and in-hospital death. A total of 866 consecutive STEMI patients underwent emergent percutaneous coronary intervention between July 2011 and June 2015. Of these 866 patients, 507 were enrolled in our study. The 507 patients were divided into 3 groups according to the initial angiograms: 1VD group, 294 patients; 2VD group, 110 patients; and 3VD/left main trunk disease (LMTD) group, 103 patients (Figure 1). The LMTD group included LMTD patients with or without any other VD. One culprit lesion was counted as one VD, and the non-culprit VD was defined as more than $75 \%$ stenosis in one angiographic view or more than $50 \%$ stenosis in two or more angiographic views.

To assess the inter-observer variability, the angiographic diagnosis of the extent of VD or LMTD required an independent view and the agreement of 2 independent cardiologists (T.S. and K.K.) who were blinded to the clinical and procedural data. Furthermore, 1 of the 2 observers (T.S.) evaluated all the angiograms again after the initial evaluation to assess the inter-observer variability for the diagnosis of the extent of VD or LMTD. In cases of disagreement, the evaluation of a third observer (H.S.) was obtained and the final decision on the diagnosis of the extent of VD or LMTD was made by consensus.

This study was conducted with the approval of the institutional ethics committee (approval no. 2059), and we obtained informed consent from all enrolled study patients to participate in this clinical study.

Data collection and laboratory examinations: Laboratory data including data for serum PUFA levels, lipid profiles, hemoglobin A1c (HbA1c), and other biochemical markers were collected routinely within 8 days after admission. Data that were unavailable within 8 days after admission were collected before discharge. Serum levels of PUFAs, including those of EPA, DHA, and AA were assayed by gas chromatography at an external laboratory (SRL, Tokyo). Lipid profiles included low-density lipoprotein cholesterol (LDL-C), high-density lipoprotein cholesterol (HDL-C), and triglycerides. The estimation of HbA1c level was based on the method certified by the National Glycohemoglobin Standardization Program. The estimated glomerular filtration rate (eGFR) was calculated by the Japanese equation using serum creatinine level, age, and sex as follows: eGFR (mL/minute $\left./ 1.73 \mathrm{~m}^{2}\right)=$ $194 \times \mathrm{Cr}^{-1.094} \times \operatorname{age}^{-0.287}(\times 0.739$ for women $) .{ }^{16)}$

Other variables: We checked all medications before the onset of STEMI, including statins, antihypertensive agents, antiplatelet agents, and antidiabetic agents. Antihypertensive agents included angiotensin-converting enzyme inhibitors, angiotensin II receptor blockers, calcium channel blockers, and $\beta$ blockers. Antiplatelet agents included aspirin, clopidogrel, ticlopidine, and cilostazol. Prasugrel and ticagrelor had not been approved in Japan during the study period.

Statistical analysis: Continuous, normally distributed data are expressed as the mean $\pm \mathrm{SD}$, and skewed data are expressed as the median and the first and third quartiles. 
Table I. Baseline Patient Characteristics

\begin{tabular}{|c|c|c|c|c|c|}
\hline & $\begin{array}{c}\text { Overall } \\
\text { (507 patients) }\end{array}$ & $\begin{array}{c}\text { 1VD } \\
\text { (294 patients) }\end{array}$ & $\begin{array}{c}\text { 2VD } \\
\text { (110 patients) }\end{array}$ & $\begin{array}{l}\text { 3VD+LMTD } \\
\text { (103 patients) }\end{array}$ & $P$ \\
\hline \multicolumn{6}{|l|}{ General characteristics } \\
\hline Age, years & $68.1 \pm 12.1$ & $66.9 \pm 12.6$ & $69.1 \pm 10.7$ & $70.4 \pm 12.0$ & 0.02 \\
\hline Men & $384(75.7)$ & $222(75.5)$ & $83(75.5)$ & $79(76.7)$ & 0.97 \\
\hline Body mass index, $\mathrm{kg} / \mathrm{m}^{2}$ & $23.9 \pm 3.5$ & $23.9 \pm 3.6$ & $24.1 \pm 3.5$ & $23.5 \pm 3.0$ & 0.46 \\
\hline Cardiogenic shock on arrival & $48(9.5)$ & $18(6.1)$ & $7(6.4)$ & $23(22.3)$ & $<0.0001$ \\
\hline Culprit Vessels & & & & & $<0.0001$ \\
\hline LAD & $242(47.7)$ & $155(52.7)$ & $48(43.6)$ & $39(37.9)$ & \\
\hline LCX & $49(9.7)$ & $24(8.2)$ & $15(13.6)$ & $10(9.7)$ & \\
\hline LMT & $9(1.8)$ & $0(0)$ & $0(0)$ & $9(8.7)$ & \\
\hline $\mathrm{RCA}$ & $207(40.8)$ & $115(39.1)$ & $47(42.7)$ & $45(43.7)$ & \\
\hline \multicolumn{6}{|l|}{ Risk Factors } \\
\hline Diabetes mellitus & $160(31.6)$ & $84(28.6)$ & $35(31.8)$ & $41(39.8)$ & 0.11 \\
\hline Hyperlipidemia & $225(44.4)$ & $130(44.2)$ & $43(39.1)$ & $52(50.5)$ & 0.25 \\
\hline Hypertension & $317(62.5)$ & $176(59.9)$ & $74(67.3)$ & $67(65.1)$ & 0.33 \\
\hline Current smoker & $179(35.3)$ & $111(37.8)$ & $37(33.6)$ & $31(30.1)$ & 0.34 \\
\hline Family history & $58(11.4)$ & $38(12.9)$ & $13(11.8)$ & $7(6.8)$ & 0.21 \\
\hline Hemodialysis & $5(1.0)$ & $1(0.3)$ & $2(1.8)$ & $2(1.9)$ & 0.22 \\
\hline \multicolumn{6}{|l|}{ Medications before admission } \\
\hline Statins & $104(20.5)$ & $58(19.7)$ & $25(22.7)$ & $21(20.4)$ & 0.80 \\
\hline Antihypertensive agents & $218(43.0)$ & $118(40.1)$ & $51(46.4)$ & $49(47.6)$ & 0.31 \\
\hline Antiplatelet agents & $51(10.1)$ & $29(9.9)$ & $8(7.3)$ & $14(13.6)$ & 0.31 \\
\hline Antidiabetic agents & $97(19.1)$ & $50(17.0)$ & $20(18.2)$ & $27(26.2)$ & 0.13 \\
\hline No medication & $204(40.2)$ & $131(44.6)$ & $40(36.4)$ & $33(32.0)$ & 0.052 \\
\hline \multicolumn{6}{|l|}{ Laboratory data } \\
\hline LDL-cholesterol, mg/dL & $100.7 \pm 31.4$ & $103.3 \pm 31.7$ & $97.5 \pm 29.9$ & $96.6 \pm 31.6$ & 0.08 \\
\hline HDL-cholesterol, mg/dL & $38.8 \pm 9.5$ & $39.3 \pm 10.1$ & $37.3 \pm 8.7$ & $37.0 \pm 8.2$ & 0.005 \\
\hline Triglycerides, mg/dL & $110.1 \pm 46.2$ & $110.1 \pm 46.2$ & $109.6 \pm 46.7$ & $110.7 \pm 45.9$ & 0.98 \\
\hline Hemoglobin A1c, \% & $5.8(5.5-6.5)$ & $5.8(5.5-6.3)$ & $5.8(5.5-6.5)$ & $6.0(5.6-6.8)$ & 0.049 \\
\hline Creatinine, mg/dL & $0.81(0.69-1.0)$ & $0.80(0.68-0.96)$ & $0.82(0.69-1.01)$ & $0.87(0.72-1.1)$ & 0.03 \\
\hline eGFR & $69.2 \pm 23.0$ & $71.4 \pm 22.5$ & $68.0 \pm 20.5$ & $64.4 \pm 26.1$ & 0.02 \\
\hline $\mathrm{BNP}, \mathrm{pg} / \mathrm{mL}$ & $116.5(45.4-266.4)$ & $97.5(39.5-201.7)$ & $145.1(56.1-302.9)$ & $188.2(54-463.3)$ & 0.0002 \\
\hline Hemoglobin, g/dL & $12.7 \pm 1.9$ & $12.9 \pm 1.9$ & $12.6 \pm 2.0$ & $12.3 \pm 1.9$ & 0.03 \\
\hline $\max \mathrm{CPK}, \mathrm{IU} / \mathrm{L}$ & $2747(1638-4416)$ & $2839(1578.3-4411.5)$ & $2824(1843.3-4323.8)$ & $2464(1605-4974)$ & 0.80 \\
\hline
\end{tabular}

Values are means (standard deviations) or medians (interquartile ranges) and are expressed as numbers (\%) unless otherwise specified. Differences between the three groups were calculated by the one-way ANOVA test. LAD indicates left anterior descending coronary artery; LCX, left circumflex coronary artery; LMT, left main trunk; RCA, right coronary artery; LDL, low-density lipoprotein; HDL, high-density lipoprotein; eGFR, estimated glomerular filtration rate; BNP, brain natriuretic peptide; and CPK, creatine phosphokinase.

Continuous variables were compared using the one-way ANOVA test or the Kruskal-Wallis test based on the distribution. Categorical variables were compared using the chi-square test or Fisher's exact test, as appropriate. Serum levels of PUFAs in the 3 groups were compared using the Bonferroni correction for multiple comparisons. Basically, $P$-values of $<0.05$ were considered to be statistically significant. By using the Bonferroni correction, $P$ values of $<0.016$ were considered to be statistically significant. Multivariate logistic regression analysis was performed to identify independent risk factors for 3VD/ LMTD. Variables for multivariable analyses were selected if they were empirically known to have an influence on atherosclerosis. The cut-off value of age was set to 70 years in accordance with daily practice, and that of eGFR was set to $60 \mathrm{~mL} /$ minute $/ 1.73 \mathrm{~m}^{2}$ to select patients having moderate to severe chronic kidney disease. Independent variables are expressed as the odds ratio with the $95 \%$ confidence interval. JMP 9 (SAS Institute Inc., Cary, NC, USA) was used for all statistical calculations.

\section{Results}

Study sample: The baseline patient characteristics are shown in Table I. There were no significant differences between the 3 groups in risk factors and in medications before admission. The extent of VD showed correlations with HDL-C, HbA1c, eGFR, brain natriuretic peptide, hemoglobin, and serum EPA level. Correlations of the extent of VD with age and cardiogenic shock were also found.

A total of $480(94.7 \%)$ of the 507 patients underwent in-hospital statin therapy.

Serum PUFA ratios by sex and age groups: The ratios of serum EPA/AA and DHA/AA varied significantly in the male patients according to the age groups and were lower in younger men but did not vary significantly in the female patients according to the age groups (Figure 2).

Serum levels of PUFAs and extent of VD: Serum EPA level in the $3 \mathrm{VD} / \mathrm{LMTD}$ group was significantly lower than that in the 1VD group $(55.5 \pm 22.1$ versus $66.2 \pm$ 28.7, $P=0.002$ ) and tended to be lower than that in the 2 VD group $(55.5 \pm 22.1$ versus $65.2 \pm 28.9, P=0.0167)$ 
EPA/AA ratio

- Male $\quad P<0.0001$

Female $P=0.37$

\section{DHA/AA ratio}

Male $P<0.0001$

Female $P=0.38$
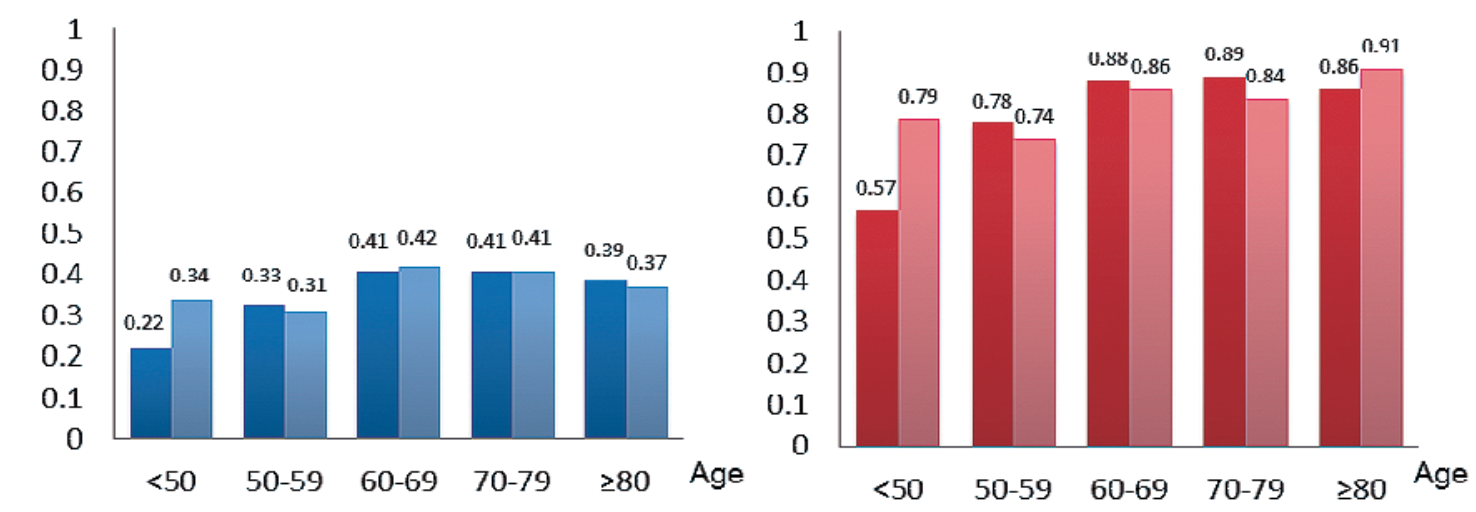

\begin{tabular}{|c|c|c|c|c|c|}
\hline Male (n) & 41 & 44 & 122 & 92 & 27 \\
\hline Fcmalc (n) & 3 & 8 & 15 & 30 & 50 \\
\hline
\end{tabular}

\begin{tabular}{|c|c|c|c|c|c|}
\hline Male (n) & 41 & 44 & 122 & 92 & 27 \\
\hline Fcmalc (n) & 3 & 8 & 15 & 30 & 50 \\
\hline
\end{tabular}

Figure 2. Ratios of serum polyunsaturated fatty acids by sex and age groups. EPA indicates eicosapentaenoic acid; DHA, docosahexaenoic acid; and AA, arachidonic acid.
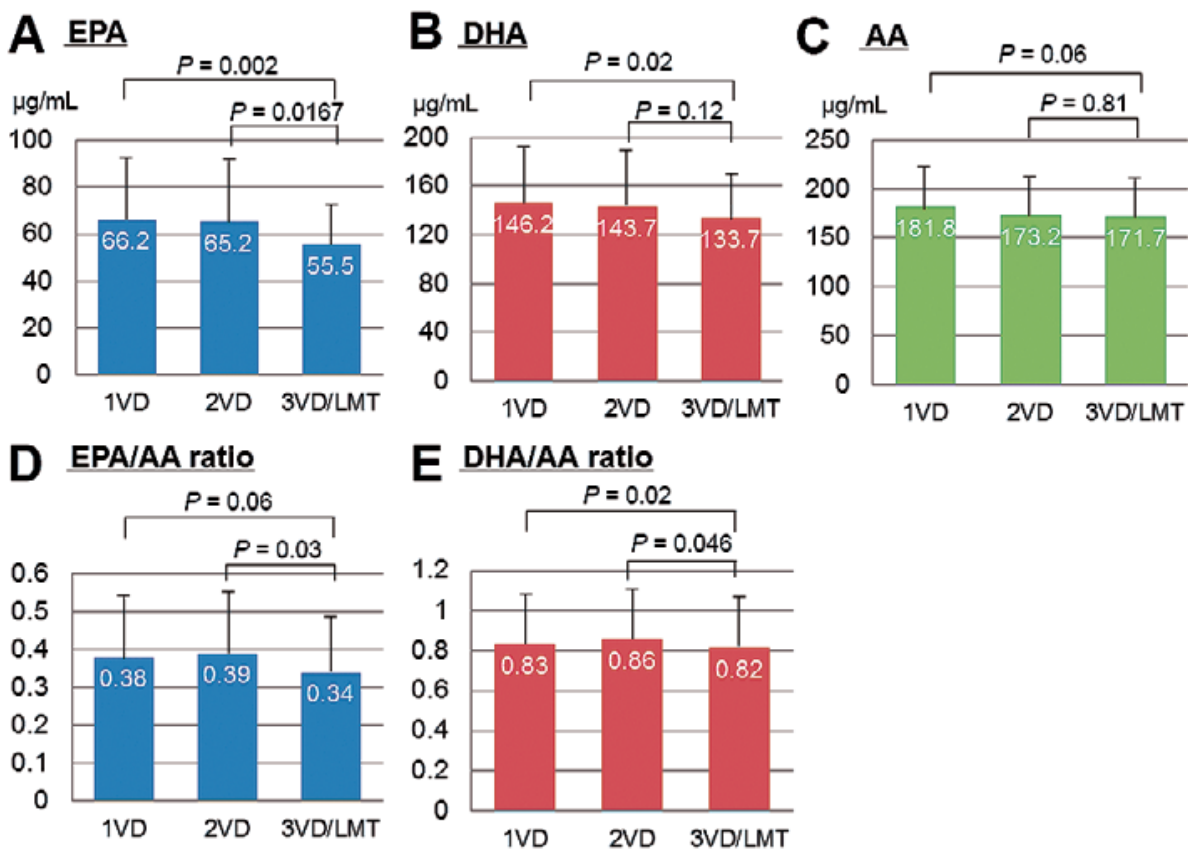

Figure 3. Relationship between serum polyunsaturated fatty acids and extent of vessel disease in patients with ST elevation myocardial infarction. A: EPA, B: DHA, C: AA, D: EPA/AA ratio, E: DHA/AA ratio. Differences between the 3 groups were calculated by the one-way ANOVA test, and Bonferroni's correction was used as a post-hoc analysis $(P<0.016)$. See the legend of Figure 2 for the definitions of abbreviations.

(Figure 3A). Serum DHA level tended to be lower in the $3 \mathrm{VD} / \mathrm{LMTD}$ group than in the $1 \mathrm{VD}$ group $(133.7 \pm 38.7$ versus $146.2 \pm 43.8, P=0.02$ ) (Figure $3 \mathrm{~B}$ ). Serum AA level was not significantly different between the 3 groups (Figure 3C). The ratios of serum EPA/AA and DHA/AA were not significantly different between the 3 groups (Figure $3 \mathrm{D}, \mathrm{E})$.

In addition, between patients with 3VD and those with LMTD, the serum EPA (55.9 versus 54.3, $P=0.75$ ), serum DHA (136.6 versus 126.2, $P=0.27$ ), and serum AA levels $(171.1$ versus $173.3, P=0.82)$ were similar. Independent risk factors for 3VD/LMTD by multivariable analysis: Multivariable analysis showed that age $\geq$ 70 years (odds ratio, 1.70; 95\% confidential interval, 1.022.86; $P=0.041$ ) and a low serum EPA level (odds ratio, $0.98 ; 95 \%$ confidential interval, $0.97-1.00 ; P=0.023$ ) 
Table II. Independent Determinants of 3VD/LMTD by Multivariable Analysis

\begin{tabular}{lccl}
\hline \multicolumn{1}{c}{ Variable } & Odds ratio & $\begin{array}{c}95 \% \text { confidence } \\
\text { interval }\end{array}$ & $P$ \\
\hline Age $\geq 70$ & 1.76 & $1.05-2.97$ & 0.03 \\
Diabetes mellitus & 1.36 & $0.82-2.26$ & 0.23 \\
HDL-C & 0.98 & $0.96-1.01$ & 0.24 \\
Statin before admission & 1.08 & $0.59-1.92$ & 0.79 \\
eGFR $\leq 60$ & 1.31 & $0.77-2.19$ & 0.32 \\
EPA & 0.98 & $0.97-1.00$ & 0.024 \\
DHA & 1.00 & $0.99-1.01$ & 0.94 \\
\hline
\end{tabular}

3VD indicates 3-vessel disease; LMTD, left main trunk disease; HDL-C, high-density lipoprotein cholesterol; eGFR, estimated glomerular filtration rate; EPA, eicosapentaenoic acid; and DHA, docosahexaenoic acid.

were independent risk factors for 3VD/LMTD, while a low serum DHA level was not (Table II).

\section{Discussion}

Our data demonstrated that the serum EPA level was inversely associated with the extent of VD in STEMI patients. Whether the serum DHA level is inversely associated with atherosclerosis is still controversial, ${ }^{8,9,17,18)}$ but multivariable analysis of the data in our study revealed that it was not associated with the extent of VD.

Because the effects of serum EPA are opposite to those of serum AA, the ratios of serum EPA/AA and DHA/AA have been reported to be correlated with inflammation in the human body and to be associated with cardiovascular events. ${ }^{5,9,19-21)}$ In our study, however, they were not significantly different between the 3 groups, which may be attributed to the distribution of the serum AA level as shown in Figure 3C. We speculate that the dietary habits of the patients affect the distribution of the serum AA level. Because the average age of patients with 1VD was significantly younger, they are likely to have more Western-style meals which often include more AA. However, it remains unclear due to the lack of data on dietary habits of the study sample before the onset of STEMI.

Serum levels of PUFAs are known to be differently distributed according to sex and age, reflecting dietary habits and age-related changes, and residential areas with different levels of fish consumption, ${ }^{22-26)}$ but their tendencies in STEMI patients remain unclear. In our study, the ratios of serum EPA/AA and DHA/AA in STEMI patients significantly varied in the male patients according to the age groups and were lower in younger men, as is the case for healthy people. ${ }^{22)}$ The distribution of the ratios of serum PUFAs was not affected by the occurrence of STEMI. The fact that the ratios of serum EPA/AA and DHA/AA did not significantly vary in the female patients according to the age groups can be attributed to the small number of women. Because serum levels of PUFAs may be affected by regional characteristics, this tendency may be peculiar to the area around our hospital.

Some randomized controlled trials have shown that supplementation of serum PUFAs may reduce cardiovascular events even during statin therapy, ${ }^{27,28)}$ but other trials have shown that such supplementation may not reduce cardiovascular events. ${ }^{29,30)}$ The discrepancy in the results of those trials may be due to differences in the amount of EPA contained in supplementation and the study patients. Administration of sufficient amounts of highly purified EPA may be beneficial for patients with severe coronary lesions.

Limitations: Our study has the following major limitations: 1) It was a single-center, cross-sectional study, 2) some patients with $3 \mathrm{VD} / \mathrm{LMTD}$ might have been excluded from the study sample due to the exclusion of in-hospital deaths, 3) lipid profiles might have been influenced by inhospital statin therapy because the timing of laboratory data collection was not on admission, 4) 3VD patients were combined with LMTD patients, 5) dietary habits before admission were not taken into consideration, and 6) we have evaluated vessel disease based on the severity of coronary artery stenosis, including both culprit and nonculprit lesions.

It remains to be studied whether administration of sufficient amounts of highly purified EPA is beneficial to patients with severe coronary lesions.

\section{Conclusion}

Low serum EPA levels may be more strongly related than low serum DHA levels to the extent of VD in STEMI patients. Age $\geq 70$ years and a low serum EPA level may be independent risk factors for $3 \mathrm{VD} / \mathrm{LMTD}$.

\section{Acknowledgments}

The authors thank Miho Kobayashi, Makiko Kanaike, and Yoshimi Sano for their secretarial assistance.

\section{Disclosures}

Conflicts of interest: Takenobu Shimada received speaking fees from Mochida Pharmaceutical Co., Ltd., Tokyo, Japan. Other authors declare that they have no competing interests.

\section{References}

1. Daviglus ML, Stamler J, Orencia AJ, et al. Fish consumption and the 30-year risk of fatal myocardial infarction. N Engl J Med 1997; 336: 1046-53.

2. Kromhout D, Bosschieter EB, de Lezenne Coulander C. The inverse relation between fish consumption and 20-year mortality from coronary heart disease. N Engl J Med 1985; 312: 1205-9.

3. Iso H, Kobayashi M, Ishihara J, et al. Intake of fish and $\mathrm{n} 3$ fatty acids and risk of coronary heart disease among Japanese: the Japan Public Health Center-Based (JPHC) Study Cohort I. Circulation 2006; 113: 195-202.

4. Tani S, Takahashi A, Nagao K, Hirayama A. Association of fish consumption-derived ratio of serum n-3 to n-6 polyunsaturated fatty acids and cardiovascular risk with the prevalence of coronary artery disease. Int Heart J 2015; 56: 260-8.

5. Ninomiya T, Nagata M, Hata J, et al. Association between ratio of serum eicosapentaenoic acid to arachidonic acid and risk of cardiovascular disease: the Hisayama Study. Atherosclerosis 2013; 231: 261-7. 
6. Adkins Y, Kelley DS. Mechanisms underlying the cardioprotective effects of omega-3 polyunsaturated fatty acids. J Nutr Biochem 2010; 21: 781-92. (Review).

7. Calder PC. The role of marine omega-3 (n-3) fatty acids in inflammatory processes, atherosclerosis and plaque stability. Mol Nutr Food Res 2012; 56: 1073-80. (Review).

8. Masson S, Marchioli R, Mozaffarian D, et al. Plasma n-3 polyunsaturated fatty acids in chronic heart failure in the GISSIHeart Failure Trial: relation with fish intake, circulating biomarkers, and mortality. Am Heart J 2013; 165: 208-15.

9. Nishizaki Y, Shimada K, Tani S, et al. Significance of imbalance in the ratio of serum $n-3$ to $n-6$ polyunsaturated fatty acids in patients with acute coronary syndrome. Am J Cardiol 2014; 113: 441-5.

10. Arakawa K, Himeno H, Kirigaya J, et al. Impact of n-3 polyunsaturated fatty acids in predicting ischemia/reperfusion injury and progression of myocardial damage after reperfusion in patients with ST-segment elevation acute myocardial infarction. J Cardiol 2015; 66: 101-7.

11. Ishii H, Murohara T. Protective effects of n-3 polyunsaturated fatty acids levels in patients with acute myocardial infarction A new target to prevent reperfusion injury. J Cardiol 2015; 66: 97-8.

12. Nodera M, Suzuki H, Yamada S, et al. Association of serum n3/n-6 polyunsaturated fatty acid ratio with T-wave alternans in patients with ischemic heart disease. Int Heart J 2015; 56: 6137.

13. Amano $\mathrm{T}$, Matsubara $\mathrm{T}$, Uetani $\mathrm{T}$, et al. Impact of omega- 3 polyunsaturated fatty acids on coronary plaque instability: an integrated backscatter intravascular ultrasound study. Atherosclerosis 2011; 218: 110-6.

14. Urabe Y, Yamamoto H, Kitagawa $\mathrm{T}$, et al. Association between serum levels of n-3 polyunsaturated fatty acids and coronary plaque detected by coronary computed tomography angiography in patients receiving statin therapy. Circ J 2013; 77: 2578-85.

15. Thygesen K, Alpert JS, Jaffe AS, et al. Third universal definition of myocardial infarction. Circulation 2012; 126: 2020-35.

16. Matsuo S, Imai E, Horio $\mathrm{M}$, et al. Revised equations for estimated GFR from serum creatinine in Japan. Am J Kidney Dis 2009; 53: 982-92.

17. Erkkilä AT, Matthan NR, Herrington DM, Lichtenstein AH. Higher plasma docosahexaenoic acid is associated with reduced progression of coronary atherosclerosis in women with CAD. J Lipid Res 2006; 47: 2814-9.

18. Mozaffarian D, Lemaitre RN, King IB, et al. Plasma phospholipid long-chain omega-3 fatty acids and total and cause-specific mortality in older adults: a cohort study. Ann Intern Med 2013;
158: 515-25.

19. Itakura H, Yokoyama M, Matsuzaki M, et al. Relationships between plasma fatty acid composition and coronary artery disease. J Atheroscler Thromb 2011; 18: 99-107.

20. Ueeda M, Doumei T, Takaya Y, et al. Association of serum levels of arachidonic acid and eicosapentaenoic acid with prevalence of major adverse cardiac events after acute myocardial infarction. Heart Vessels 2011; 26: 145-52.

21. Domei T, Yokoi H, Kuramitsu S, et al. Ratio of serum n-3 to n6 polyunsaturated fatty acids and the incidence of major adverse cardiac events in patients undergoing percutaneous coronary intervention. Circ J 2012; 76: 423-9.

22. Yanagisawa N, Shimada K, Miyazaki T, et al. Polyunsaturated fatty acid levels of serum and red blood cells in apparently healthy Japanese subjects living in an urban area. J Atheroscler Thromb 2010; 17: 285-94.

23. de Groot RH, van Boxtel MP, Schiepers OJ, Hornstra G, Jolles J. Age dependence of plasma phospholipid fatty acid levels: potential role of linoleic acid in the age-associated increase in docosahexaenoic acid and eicosapentaenoic acid concentrations. $\mathrm{Br}$ J Nutr 2009; 102: 1058-64.

24. Saga LC, Liland KH, Leistad RB, Reimers A, Rukke EO. Relating fatty acid composition in human fingertip blood to age, gender, nationality and n-3 supplementation in the Scandinavian population. Int J Food Sci Nutr 2012; 63: 790-5.

25. Kitagawa Y, Abe S, Toyoda S, et al. Gender differences in the ratio of eicosapentaenoic acid to arachidonic acid in an inland prefecture, Tochigi: Tochigi Ryomo EPA/AA Trial in Coronary Artery Disease (TREAT-CAD). Intern Med 2014; 53: 177-82.

26. Nakamura T, Azuma A, Kuribayashi T, Sugihara H, Okuda S, Nakagawa M. Serum fatty acid levels, dietary style and coronary heart disease in three neighbouring areas in Japan: the Kumihama study. Br J Nutr 2003; 89: 267-72.

27. Yokoyama M, Origasa H, Matsuzaki M, et al. Effects of eicosapentaenoic acid on major coronary events in hypercholesterolaemic patients (JELIS): a randomised open-label, blinded endpoint analysis. Lancet 2007; 369: 1090-8.

28. Tavazzi L, Maggioni AP, Marchioli R, et al. Effect of n-3 polyunsaturated fatty acids in patients with chronic heart failure (the GISSI-HF trial): a randomised, double-blind, placebo-controlled trial. Lancet 2008; 372: 1223-30.

29. Bosch J, Gerstein HC, Dagenais GR, et al. n-3 fatty acids and cardiovascular outcomes in patients with dysglycemia. N Engl J Med 2012; 367: 309-18.

30. Risk and Prevention Stdy Collaborative Group. n-3 fatty acids in patients with multiple cardiovascular risk factors. N Engl J Med 2013; 368: 1800-8. 